Homology, Homotopy and Applications, vol.3, No.2, 2001, pp.37-53

\title{
CHAIN FUNCTORS WITH ISOMORPHIC HOMOLOGY
}

\author{
FRIEDRICH W. BAUER
}

(communicated by Walter Tholen)

\begin{abstract}
Every chain functor $\mathbf{K}_{*}$ determines a homology theory on a given category of topological spaces resp. of spectra $H_{*}\left(\mathbf{K}_{*}\right)(\cdot)$ cf. $\S 4$. If $\mathbf{K}_{*}, \mathbf{L}_{*}$ are chain functors such that $H_{*}\left(\mathbf{K}_{*}\right)(\cdot) \approx H_{*}\left(\mathbf{L}_{*}\right)(\cdot)$ then there exists a third chain functor $\mathbf{C}_{*}$ and transformations of chain functors ${ }^{K} \gamma: \mathbf{K}_{*} \longrightarrow \mathbf{C}_{*}$, ${ }^{L} \gamma: \mathbf{L}_{*} \longrightarrow \mathbf{C}_{*}$ inducing isomorphisms of the associated homology theories (theorem 1.1.). Moreover the distinction between regular and irregular chain functors is introduced.
\end{abstract}

\section{Introduction}

Let $h_{*}()=\left\{h_{n}(), \partial, n \in \mathbb{Z}\right\}$ be a homology theory, given on a category of pairs of topological spaces or pairs of spectra (cf. [8]). A chain theory associated with $h_{*}$ consists of a functor

$$
K_{*}: \mathfrak{K} \longrightarrow \mathbf{c h} \text { (= category of chain complexes), }
$$

and natural short exact sequences

$$
0 \longrightarrow K_{*}(A) \stackrel{i_{*}}{\longrightarrow} K_{*}(X) \stackrel{j_{*}}{\longrightarrow} K_{*}(X, A) \longrightarrow 0
$$

such that $H_{*}\left(K_{*}(X, A)\right)$ is naturally isomorphic to $h_{*}(X, A)$, and that this isomorphism is compatible with the boundary operator

$$
\partial: H_{n}\left(K_{*}(X, A)\right) \longrightarrow H_{n-1}\left(K_{*}(A)\right)
$$

induced by (2).

The main result of $[6]$ is, that for CW spaces, the existence of such a chain theory is equivalent to the property that $h_{*}$ is the direct sum of ordinary homology theories. In order to find some appropriate substitute for chain theories (which do not exist for arbitrary homology theories) chain functors were introduced ( for the first time in $[2])$ :

A chain functor $\boldsymbol{K}_{*}=\left\{K_{*}, K_{*}^{\prime}, i^{\prime}, l, \varphi, \kappa\right\}$ consists of a functor

$$
K_{*}: \mathfrak{K} \longrightarrow \mathbf{c h} \text { (= category of chain complexes), }
$$

Received February 24, 2000, revised November 23, 2000; published on February 3, 2001.

2000 Mathematics Subject Classification: 55N20, 18G35, 55N39.

Key words and phrases: regular and irregular-chain-functors, homology theories associated with chain functors.

(c) 2001, Friedrich W. Bauer. Permission to copy for private use granted. 
$\mathfrak{K}$ a suitable category of pairs of topological spaces or of spectra, a subfunctor $l: K_{*}^{\prime} \subset K_{*}$, a natural inclusion $i^{\prime}: K_{*}(A) \subset K_{*}^{\prime}(X, A)$ together with some other structural (non-necessary natural) mappings $\varphi: K_{*}^{\prime}(X, A) \longrightarrow K_{*}(X), \kappa$ : $K_{*}(X) \longrightarrow K_{*}^{\prime}(X, A)$, satisfying certain properties.

Every homology theory $h_{*}()=\left\{h_{n}(), \partial, n \in \mathbb{Z}\right\}$ admits a chain functor such that the derived homology homology $H_{*}\left(K_{*}(X, A)\right)$ is naturally isomorphic to $h_{*}(X, A)$. In addition the boundary

$$
\partial: h_{n}(X, A) \longrightarrow h_{n-1}(A)
$$

is determined by the chain functor $\boldsymbol{K}_{*}$.

It turns out that every chain theory (i.e. every functor (1), accompanied by a natural exact sequence (2)) determines to a chain functor, but not vice-versa. The question, under what conditions a chain functor gives rise to a homology theory, which is associated with a chain theory, has something to do with the non-naturality of $\kappa$ and $\varphi$ (cf. $\S 5$ for further references).

Concerning details about chain functors, the reader is referred to $\S 4$, resp. to $\S 5$ concerning the motivation. Apart from recalling the definition of a chain functor, the purpose of $\S 4$ is the introduction of the stronger concept of a regular and the weaker concept of an irregular-chain-functor (definition 4.1.) as well as some material about transformations of chain functors (propositions 4.4., 4.5.). All this is needed in [1].

The derived homology of an irregular-chain-functor does not furnish a homology theory; irregular-chain-functor cannot be realized as spectra. Nevertheless, they are needed to formulate some of the results about tensor produts of chain functors and spectra in [1]. The existence theorem of a chain functor for a given homology theory is formulated for homology theories, which are defined on a category of pairs of spectra (in our case: CW-spectra, hence objects of the Boardman category) rather than on a category of pairs of spaces (cf. [8] ).

Every homology theory $h_{*}$ is associated to a chain functor (i.e. the derived homology of this chain functor is isomorphic to $h_{*}$ ). Therefore the following quite natural question arises:

Let $\mathbf{K}_{*}, \mathbf{L}_{*}$ be two chain functors with isomorphic associated homology (i.e. there is an isomorphism of homology theories $\left.\psi: H_{*}\left(\mathbf{K}_{*}\right) \approx H_{*}\left(\mathbf{L}_{*}\right)\right)$ What can be said about the relationship between $\mathbf{K}_{*}$ and $\mathbf{L}_{*}$ ? It can not be expected that they are isomorphic as chain functors. However theorem 1.1. asserts that there exists a chain functor $\mathbf{C}_{*}$ and transformations

$$
\mathbf{K}_{*} \stackrel{{ }^{K} \gamma}{\longrightarrow} \mathbf{C}_{*} \stackrel{{ }^{L} \gamma}{\longleftarrow} \mathbf{L} *
$$

inducing isomorphisms of the associated homology such that

$$
\psi=\left({ }^{L} \gamma_{*}\right)^{-1} K_{*} .
$$

Apart from the general interest of this result, it is needed in a forthcoming paper for the definition of the tensor-product of CW-spectra (more precisely: for the verification of the independence of this construction of the choice of the chain functors involved). 
The proof of theorem 1.1. is displayed in $\S 1-\S 3$.

Results about chain functors are recorded in the expository article [6].

\section{The Main Theorem}

Let $\mathbf{A}_{*}, \mathbf{B}_{*}$ be two chain functors and $\psi: H_{*}\left(\mathbf{A}_{*}\right) \approx H_{*}\left(\mathbf{B}_{*}\right)$ an isomorphism of the associated homology theories.

We do not necessarily find a mapping $\lambda: \mathbf{A}_{*} \longrightarrow \mathbf{B}_{*}$ inducing $\psi$. However we are able to prove:

1.1.Theorem: There exists a chain functor $\mathbf{C}_{*}$ and mappings of chain functors

$$
\mathbf{A}_{*} \stackrel{{ }^{A} \gamma}{\longrightarrow} \mathbf{C}_{*} \stackrel{{ }^{B} \gamma}{\longleftarrow} \mathbf{B}_{*}
$$

inducing isomorphism of homology theories ${ }^{B} \gamma_{*},{ }^{B} \gamma_{*}$ such that

$$
\left({ }^{B} \gamma_{*}\right)^{-1}{ }^{A} \gamma_{*}=\psi
$$

It turns out that the mapping of chain functors ${ }^{A} \gamma\left({ }^{B} \gamma\right)$ appears as the composition of two mappings of chain functors

$$
\begin{aligned}
& \mathbf{A}_{*} \stackrel{{ }^{A} \lambda}{\longrightarrow}{ }^{s} \mathbf{A}_{*} \stackrel{{ }^{A} \eta}{\longrightarrow} \mathbf{C}_{*} \\
& \mathbf{B}_{*} \stackrel{{ }^{B} \lambda}{\longrightarrow}{ }^{s} \mathbf{B}_{*} \stackrel{{ }^{B} \eta}{\longrightarrow} \mathbf{C}_{*},
\end{aligned}
$$

where ${ }^{s} \mathbf{A}_{*}\left({ }^{s} \mathbf{B}_{*}\right)$ have a special property:

1.2. Definition: A chain functor $\mathbf{K}_{*}$ is a $\mathrm{s}$-chain functor whenever the following holds:

(*) There exists an (not necessarily additive) assignment which assigns to each $\zeta \in H_{n}\left(K_{*}(X, A)\right)$ a cycle $z=z(\zeta) \in \zeta$ (i.e. $[z]=\zeta$ ) such that for any $f$ : $(X, A) \longrightarrow(Y, B)$ in $\mathfrak{K}^{2}$ (the category on which the chain functor is defined) one has:

$$
f_{\#} z(\zeta)=z\left(f_{*}(\zeta)\right)
$$

where we denote as usual: $f_{*}=H_{*}\left(K_{*}\right)(f), f_{\#}=\mathbf{K}_{*}(f)$.

The main tool for proving theorem 1.1. is:

1.3. Proposition: To each chain functor $\mathbf{A}_{*}$ there exists a s-chain functor ${ }^{s} \mathbf{A}_{*}$ and a mapping of chain functors

$$
{ }^{A} \lambda: \mathbf{A}_{*} \longrightarrow{ }^{s} \mathbf{A}_{*}
$$

inducing an isomorphism of the associated homology theories. The proof is relegated to the next two sections.

Proof of theorem 1.1.: We have an isomorphism of homology theories ${ }^{s} \psi: H_{*}\left({ }^{s} \mathbf{A}_{*}\right) \stackrel{\approx}{\longrightarrow} H_{*}\left({ }^{s} \mathbf{B}_{*}\right)$ commuting with ${ }^{A} \lambda_{*},{ }^{B} \lambda_{*}$ and $\psi$.

Let ${ }^{A} \zeta \in H_{n}\left({ }^{s} \mathbf{A}\right)(X, A)$ be given, then we have ${ }^{B} \zeta={ }^{s} \psi\left({ }^{A} \zeta\right) \in H_{n}\left({ }^{s} \mathbf{B}\right)(X, A)$ 
and the assignments ${ }^{A} z\left({ }^{A} \zeta\right)={ }^{A} z \in{ }^{A} \zeta,{ }^{B} z={ }^{B} z\left({ }^{B} \zeta\right) \in{ }^{B} \zeta$, satisfying $(\star)$ in definition 1.2.

In

$$
{ }^{s} A_{*}(X, A) \oplus{ }^{s} B_{*}(X, A)=C_{*}^{(1)}(X, A)
$$

we invent new chains $x\left({ }^{A} \zeta\right)$ in dimension $n+1$, satisfying

$$
d x\left({ }^{A} \zeta\right)={ }^{A} z-{ }^{B} z
$$

Let $f:(X, A) \longrightarrow(Y, B)$ be a given mapping, then we define

$$
f_{\#} x\left({ }^{A} \zeta\right)=x\left(f_{*}\left({ }^{A} \zeta\right)\right)
$$

The chain complex $C_{*}^{(1)}$ amended by these new connecting chains $x$ is called $C_{*}^{(2)}(X, A)$. This construction is clearly functorial.

In a last step we take the subcomplex $K_{*}(X, A) \subset C_{*}^{(2)}(X, A)$ generated by all $x(\cdot)$ and form the cone over $\mathbf{K}_{*}$ :

$$
C_{*}(X, A)=C_{*}^{(2)}(X, A) \cup \text { cone } K_{*}(X, A) .
$$

Observe that a cycle in $K_{*}(X, A)$ consisting of elements $x(\cdot)$ can only appear for algebraic reasons ( being the sum of chains like e.g. $x\left(\alpha_{1}{ }^{A} \zeta_{1}+\alpha_{2}{ }^{A} \zeta_{2}\right)-\alpha_{1} x\left({ }^{A} \zeta_{1}\right)-$ $\left.\alpha_{2} x\left({ }^{A} \zeta_{2}\right), \alpha_{i} \in \mathbb{Z}\right)$. As a result all cycles in $\mathbf{C}_{*}(X, A)$ are homologous to a cycle in ${ }^{s} \mathbf{A}_{*}$ resp. in ${ }^{s} \mathbf{B}_{*}$ and such a cycle is bounding in $\mathbf{C}_{*}(X, A)$ whenever it bounds in ${ }^{s} \mathbf{A}_{*}$ (resp. in ${ }^{s} \mathbf{B}_{*}$ ).

We endow $\mathbf{C}_{*}$ with the structure of a chain functor such that there are inclusions of chain functors

$$
{ }^{A} \eta:{ }^{s} \mathbf{A}_{*} \longrightarrow \mathbf{C}_{*} \longleftarrow{ }^{s} \mathbf{B}_{*}:{ }^{B} \eta
$$

in an obvious way:

We detect $C_{*}^{\prime}$ by performing the previous constructions in ${ }^{s} A_{*}^{\prime},{ }^{s} B_{*}^{\prime}$, while the mappings $\kappa, \varphi, l, i^{\prime}$ are more or less obvious.

Moreover we deduce immediately from our construction that ${ }^{A} \eta,{ }^{B} \eta$ induce isomorphisms for the associated homology and that

$$
\left({ }^{B} \eta_{*}\right)^{-1 A} \eta_{*}={ }^{s} \psi
$$

Hence ${ }^{A} \gamma={ }^{A} \eta{ }^{A} \lambda$, resp. for $B$ satisfy the requirements of the theorem.-

\section{Preparation of the proof of proposition 1.2 .}

Before displaying the details, we give an idea of the proof:

Let $\mathbf{K}_{*}$ be a chain functor. We can assign to each homology class $\zeta \in H_{n}\left(\mathbf{K}_{*}\right)(X, A)$ a cycle $z=z(\zeta) \in \zeta$. If $f:(X, A) \longrightarrow(Y, B)$ is a mapping, then we have for the homology classes $f_{*}([z(\zeta)])=\left[z\left(f_{*}(\zeta)\right]\right.$, hence we can fix a chain $x^{(1)}=x^{(1)}(f, \zeta) \in$ $K_{*}(Y, B)$ such that

$$
d x^{(1)}=f_{\#} z(\zeta)-z\left(f_{*}(\zeta)\right) .
$$

If

$$
(X, A) \stackrel{f_{1}}{\longrightarrow}\left(X_{1}, A_{2}\right) \stackrel{f_{2}}{\longrightarrow}\left(X_{2}, A_{2}\right)
$$


are two mappings, then we fix $x^{(2)}\left(f_{1}, f_{2}, \zeta\right) \in \mathbf{K}_{*}\left(X_{2}, A_{2}\right)$ such that

$$
d x^{(2)}=x^{(1)}\left(f_{2} f_{1}, \zeta\right)-x^{(1)}\left(f_{2}, f_{1 *}(\zeta)\right)-f_{2 \#} x^{(1)}\left(f_{1}, \zeta\right) .
$$

So we continue and construct chains of increasing dimensions:

$$
x^{(k)}\left(f_{1}, \cdots, f_{k}, \zeta\right) \in \mathbf{K}_{n+k}\left(X_{k}, A_{k}\right)
$$

such that

$$
d x^{(k)}=\sum_{i=0}^{k}(-1)^{i+1} x^{(k-1)}\left(f_{1}, \cdots, f_{i} f_{i+1}, \cdots, f_{k}, \zeta\right)
$$

where for $i=k$ we take

$$
f_{k \#} x^{(k-1)}\left(f_{1}, \cdots, f_{k-1}, \zeta\right)
$$

and for $i=0$

$$
x^{(k-1)}\left(f_{2}, \cdots, f_{k}, f_{1 *} \zeta\right) .
$$

Such an assignment $\zeta \mapsto\left\{x^{(i)} \mid i=0,1, \ldots\right\}$ with $x^{(0)}(\zeta)=z(\zeta)$ is called $\omega$. In order to be able define a chain functor ${ }^{s} \mathbf{K}_{*}$ and establish a mapping of chain functors $\lambda: \mathbf{K}_{*} \longrightarrow{ }^{s} \mathbf{K}_{*}$ we are obliged to introduce pro-chains:

2.1. Definition Let $\sigma=\left\{c, x^{(k)}, \bar{x}^{(k)}, k=1,2, \cdots\right\}$ be a collection of chains $c \in$ $K_{n}(X, A), \quad x^{(k)} \in K_{n+k}(Y, B), \quad \bar{x}^{(k)} \in K_{n+k-1}(Y, B)$ for all $(X, A),(Y, B) \in \mathfrak{K}^{2}$ such that the following conditions hold:

p1)If $f:(X, A) \longrightarrow(Y, B)$ is a mapping, $c \in K_{n}(X, A) \cap \sigma$, then there exists a unique $c_{1} \in \mathbf{K}_{n}(Y, B) \cap \sigma$ and $x^{(1)}=x^{(1)}(f, c) \in K_{n+1}(Y, B) \cap \sigma, \quad \bar{x}^{(1)}=$ $\bar{x}^{(1)}(f, d c) \in K_{n}(Y, B) \cap \sigma$ such that

$$
d x^{(1)}+\bar{x}^{(1)}=f_{\#} c-c_{1} .
$$

we say that $c \leqslant c_{1}$ in $\sigma$ or $c \underset{\sigma}{\leqslant} c_{1}$.

More general: For mappings $f_{i}:\left(X_{i}, A_{i}\right) \longrightarrow\left(X_{i+1}, A_{i+1}\right)$,

$$
(X, A)=\left(X_{1}, A_{1}\right) \stackrel{f_{1}}{\longrightarrow} \cdots \stackrel{f_{k}}{\longrightarrow}\left(X_{k+1}, A_{k+1}\right)=(Y, B)
$$

assume we have

$$
x^{(j)}=x^{(j)}\left(f_{1}, \cdots, f_{j}, c_{1}, \cdots c_{j}\right) \in K_{n+j}\left(X_{j+1}, A_{j+1}\right) \quad c_{j} \in K_{n}\left(X_{j}, A_{j}\right)
$$

already defined for $j \leqslant k-1$ (correspondingly $\bar{x}^{(j)}=\bar{x}^{(j)}\left(f_{1}, \cdots, f_{j}, d c_{1}, \cdots, d c_{j}\right) \in$ $\left.K_{n+j-1}\right)$. Then we find $x^{(k)}$ such that

$$
d x^{(k)}+\bar{x}^{(k)}=\sum_{i=0}^{k}(-1)^{i+1} x^{(k-1)}\left(f_{1}, \cdots, f_{i} f_{i+1}, \cdots, f_{k}, c_{1}, \cdots, \hat{c}_{i}, \cdots, c_{k}\right)
$$

where for $i=k$ we take

$$
f_{k \#} x^{(k-1)}\left(f_{1}, \cdots, f_{k-1}, c_{1}, \cdots, c_{k-1}\right)
$$

and for $i=0$

$$
x^{(k-1)}\left(f_{2}, \cdots, f_{k}, c_{2}, \cdots, c_{k}\right) .
$$


p2) To any two elements $c_{1}, \quad c_{2} \in \sigma$ there exists a $c \in \sigma, \quad c \underset{\sigma}{\leqslant} c_{i}$. Then $\sigma$ is a pro-chain in $\mathbf{K}_{*}$.

Remarks and examples: 1$)$ Let $c \in K_{n}(X, A)$ be given, then $\mathbf{c}=\left\{f_{\#} c, 0\right\}$, $f:(X, A) \longrightarrow(Y, B)$ for any $(Y, B) \in \mathfrak{K}^{2}$ is a pro-chain, which is generated by $c$.

2) Suppose that there exists in $\sigma$ a $c \in K_{n}(X, A)$ such that $c \underset{\sigma}{\leqslant} c_{1}$ for any $c_{1} \in \sigma$ Then we say that $\sigma$ is a principal pro-chain, generated by $c$. The preceding $\mathbf{c}$ is an example of a principal pro-chain.

3) Let $\sigma$ be any pro-chain, $c \in K_{n}(X, A) \cap \sigma$, then $\mathbf{c}_{\sigma}=\left\{c_{1} \mid c \underset{\sigma}{\leqslant} c_{1}\right\}$ with corresponding $x^{(i)}, \bar{x}^{(i)}$ is a principal pro-chain generated by $c$ in $\sigma$.

4) If we take a pro-chain $\sigma$ with all $x^{(i)}, \bar{x}^{(i)}=0$, then $c \leqslant c_{1}$ in $\sigma$ simply means, that there exists a $f \in M$ or $\mathfrak{K}^{2}$ with $f_{\#} c=c_{1}$. The higher conditions in p1) $(k \geqslant 2)$ are all redundant and p1), p2) means that $\sigma$ is an ideal.

5 ) The assignment $\omega$ which we introduced at the beginning of this section is a prochain; although not being an ideal, it is associated with a principal pro-chain (all $c \geqslant z(\zeta)$, where the $\leqslant-$ relation is determined by $\omega$ ).

$6)$ Let $\sigma_{1}, \sigma_{2}$ be two principal pro-chains, with generating $c_{1}, c_{2} \in K_{n}(X, A)$. Then we can add all items in both pro-chains, obtaining this way a principal pro-chain $\sigma_{1}+\sigma_{2}$ generated by $c_{1}+c_{2}$.

7) Let $\sigma=\left\{c, x^{(k)}, \bar{x}^{(k)}\right\}$, be any pro-chain, then

$$
d \sigma=\left\{d c, \bar{x}^{(k)}\right\}
$$

is again a pro-chain, the boundary of $\sigma$.

A pro-chain is a pro-cycle, whenever all $\bar{x}^{(k)}=0$, or alternatively $d \sigma$ contains only zeros. If $\sigma$ is generated by $c$, then $d \sigma$ is again a principal pro-chain, generated by $d c$.

8) Let $g:(X, A) \longrightarrow(Y, B)$ be a mapping and $\sigma$ a principal pro-chain, generated by $c \in K_{n}(X, A)$ then we find a unique $c_{1} \in K_{n}(Y, B) \cap \sigma, c \leqslant c_{1}$ and define:

$$
g_{\#} \sigma=c_{1 \sigma} .
$$

This assignment is additive and compatible with the formation of boundaries. Moreover everything is functorial.

$9)$ Let $\bar{K}_{*}(X, A)$ be the chain complex of principal pro-chains generated by all $c \in K_{*}(X, A)$ then

$$
\overline{\mathbf{K}}_{*}: \mathfrak{K}^{2} \longrightarrow \mathbf{c h}
$$

is a functor (into the category of (free) chain complexes).

The assignment

$$
c \in K_{n}(X, A) \longmapsto \mathbf{c} \in \bar{K}_{*}(X, A)
$$

of example 1) yields a natural transformation $\lambda: \mathbf{K}_{*} \longrightarrow \overline{\mathbf{K}}_{*}$.

10) Let $\sigma \in \bar{K}_{n}(X, A)$ be a principal pro-cycle, generated by $z \in K_{n}(X, A)$. Then $\sigma \sim \mathbf{z}$ in $\bar{K}_{*}(X, A)$ : The required principal pro-chain $\xi \in \bar{K}_{n+1}(X, A)$ with $d \xi=$ $\sigma-\mathbf{z}$ is generated by 0 (although not being zero as a pro-chain!) and has the

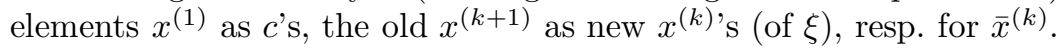

Suppose we have $d \sigma=\mathbf{z}$ in $\bar{K}_{*}(X, A)$, then we infer in the same manner, that $d \mathbf{c}=\mathbf{z}, c \in K_{n+1}(X, A)$ being the generating element of $\sigma$. 
As a result:

The natural transformation $\lambda$ induces an isomorphism in homology.

11) We fix $\omega$ as in the beginning and define for any $\zeta \in H_{n}\left(\mathbf{K}_{*}\right)(X, A)$

$$
\mathbf{z}(\zeta)=\mathbf{z}(\zeta)_{\omega}
$$

By definition we have

$$
f_{\#} \mathbf{z}(\zeta)=\mathbf{z}\left(f_{*}(\zeta)\right) .
$$

\section{Proof of proposition 1.2.}

In the preceding section we established a functor $\overline{\mathbf{K}}_{*}: \mathfrak{K}^{2} \longrightarrow$ ch which must be equipped with the structure of a chain functor: $\bar{K}_{*}^{\prime}(X, A)$ consists of all those principal pro-chains $\sigma=\left\{c, x^{(k)}, \bar{x}^{(k)}\right\}$ with $c \in K_{n}^{\prime}$. This yields a functor $\bar{K}_{*}^{\prime}$ : $\mathfrak{K}^{2} \longrightarrow \mathbf{c h}$ as well as an inclusion

$$
l: \bar{K}_{*}^{\prime}(X, A) \subset \bar{K}_{*}(X, A) .
$$

Let $\sigma^{\prime} \in \bar{K}_{*}^{\prime}(X, A)$ with generating $c^{\prime}$ be given, then we set $\varphi\left(\sigma^{\prime}\right)=\varphi\left(c^{\prime}\right)$.

Suppose $\sigma \in \bar{K}_{n}(X)$ with generating $c$ be given, then we set $\kappa(\sigma)=\kappa(c)$.

Since $\varphi \kappa \simeq 1$ in $\mathbf{K}_{*}$, we have $x: \varphi \kappa(c) \simeq c$ so that $\mathbf{x}: \varphi \kappa \simeq 1_{\sigma}$ is a chain homotopy. This provides us with non-natural chain mappings $\kappa, \varphi$ and a non-natural chain homotopy $\varphi \kappa \simeq 1$. The chain homotopy $j_{\#} \varphi \simeq l$ is treated in the same way. Let $\sigma$ be a cycle in $\bar{K}_{n}(X, A)$ then we find $z \in Z_{n}\left(K_{*}(X, A)\right), \quad z^{\prime} \in K_{n}^{\prime}(X, A)$, $a \in K_{n}(A, A)$ such that

$$
\sigma \sim \mathbf{z} \sim \mathbf{l}\left(\mathbf{z}^{\prime}\right)+\mathbf{q}_{\#}(\mathbf{a})=l\left(\mathbf{z}^{\prime}\right)+q_{\#}(\mathbf{a}) .
$$

Therefore $\psi: H_{n}\left(\overline{\mathbf{K}}_{*}^{\prime \prime}\right)(X, A) \longrightarrow H_{n}\left(\overline{\mathbf{K}}_{*}\right)(X, A)$ is epic.

The remaining properties of a chain functor are verified by the same kind of argument.

We set $\overline{\mathbf{K}}_{*}={ }^{s} \mathbf{K}_{*}$ and observe that $\lambda: \mathbf{K}_{*} \longrightarrow{ }^{s} \mathbf{K}_{*}, \quad \lambda(c)=\mathbf{c}$ is a mapping of chain functors, which, according to remark 10 in $\S 2$ induces an isomorphism in homology. Property $(\star)$ of proposition 1.2. follows from remark 11) (3) in §2.

This completes the proof of proposition 1.2.-

Remark: The conceptual background of the construction of a $s$ - chain functor goes back to K. Sitnikov's construction a Steenrod-Sitnikov homology: Instead of stating that two cycles $\left.z_{i} \in Z_{n}\left(X_{i}\right)\right), i=1,2, X_{1} \subset X_{2}$ are homologous one has to specify a chain $x_{12}$ furnishing that homology. Alternatively one can think of the construction of a strong shape category, where one must employ higher individual homotopies of arbitrary degrees (rather than just stating that two mappings are homotopic).

\section{Chain Functors:}

We recall the definition of a chain functor and of a transformation (or a mapping between) chain functors. Concerning the motivation and the main results about 
chain functors the reader is referred to $[2]-[5]$ resp. to $\S 5$.

Let $\mathfrak{K}$ be a subcategory of Top and $\mathfrak{K}^{2}$ a category of pairs $(X, A), i: A \subset X, j$ : $X \subset(X, A), A, X \in \mathfrak{K}$.

Suppose $\mathbf{C}_{*}: \mathfrak{K} \longrightarrow \mathbf{c h}$ (= category of free chain complexes with natural bases) is a functor and $\mathbf{C}_{*}^{\prime} \subset \mathbf{C}_{*}$ a subfunctor, being equipped with the following additional structures: There are functors

$$
C_{*}, C_{*}^{\prime}: \mathfrak{K}^{2} \longrightarrow \mathbf{c h},
$$

and natural inclusions $l: C_{*}^{\prime} \subset C_{*}, i^{\prime}: C_{*}(A) \subset C_{*}^{\prime}(X, A)$ such that $\mathbf{C}_{*}(X)=$ $C_{*}(X, X), \mathbf{C}_{*}^{\prime}(X)=C_{*}^{\prime}(X, X)$. We denote induced maps $C_{*}(f)$ always by $f_{\#}$.

There are (not necessarily natural) chain transformations

$$
\begin{gathered}
\varphi_{\#}: C_{*}^{\prime}(X, A) \longrightarrow C_{*}(X) \\
\kappa_{\#}: C_{*}(X) \longrightarrow C_{*}^{\prime}(X, A)
\end{gathered}
$$

together with chain homotopies resp. relations:

$$
\text { D1) } \quad\left\{\begin{array}{l}
\varphi_{\#} \kappa_{\#} \simeq 1 \\
j_{\#} \varphi_{\#} \simeq l \\
\kappa_{\#} i_{\#}=i^{\prime}, \quad i: A \subset X .
\end{array}\right.
$$

We have a diagram with exact upper row $\left(p: C_{*}^{\prime}(X, A) \longrightarrow C_{*}^{\prime \prime}(X, A)=\right.$ $C_{*}^{\prime}(X, A) / i m i^{\prime}$ being simply the projection):

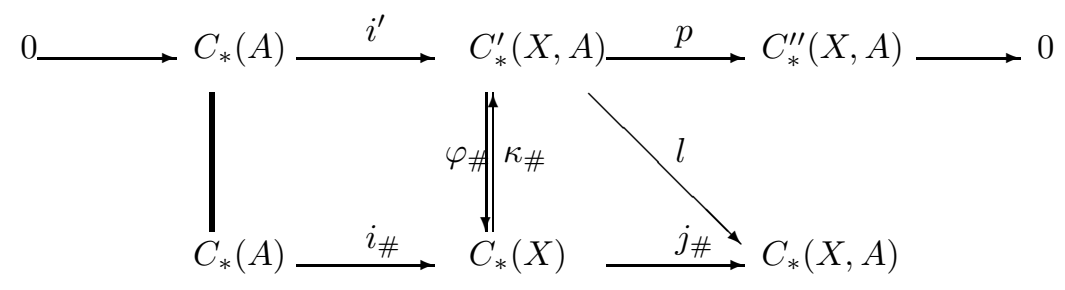

and a natural mapping

$$
\psi: H_{*}\left(C_{*}^{\prime \prime}(X, A)\right) \longrightarrow H_{*}\left(C_{*}(X, A)\right)
$$

defined by

$$
\psi\left(z^{\prime \prime}\right)=\left\{l\left(z^{\prime}\right)+q_{\#}(a)\right\}
$$

where $z^{\prime \prime}$ is the homology class of a $z^{\prime} \in C_{n}^{\prime}(X, A)$, with

$$
d z^{\prime} \in i m\left(i^{\prime}: C_{n-1}(A) \longrightarrow C_{n-1}^{\prime}(X, A)\right)
$$

and $a \in C_{n}(A, A)$,

$$
d a=-s_{\#} i^{\prime-1} d z^{\prime}
$$




$$
p\left(z^{\prime}\right)=z^{\prime \prime}, \quad q:(A, A) \subset(X, A), s: A \subset(A, A) .
$$

In view of condition $\star \star)$ below, $\psi\left(z^{\prime \prime}\right)$ depends only on $z^{\prime}$ but not on $a$. We require D2) $\psi$ is an epimorphism;

$$
\operatorname{ker} j_{*} \subset \operatorname{ker} p_{*} \kappa
$$

with

$$
\kappa=\kappa_{\# *}: H_{*}\left(C_{*}(X)\right) \longrightarrow H_{*}\left(C_{*}^{\prime}(X, A)\right), \quad f_{*}=H_{*}(f)
$$

for any $f \in \operatorname{Mor} \mathfrak{K}^{2}$.

In order to simplify the notation we will denote (by an abuse of notation) $\varphi_{\#}$ as well as $\varphi_{\# *}$ simply by $\varphi$ (resp. for $\kappa$ ) where the precise meaning will be always clear from the context.

D3) Let $\bar{\partial}: H_{n}\left(C_{*}^{\prime \prime}(X, A)\right) \longrightarrow H_{n-1}\left(C_{*}(A)\right)$ be the boundary associated with the exact row $(\bar{S})$, then we have

$$
\operatorname{ker} \psi \subset \operatorname{ker} \bar{\partial} \text {. }
$$

Moreover

*) All inclusions $f:(X, A) \subset(Y, B)$ induce monomorphisms; moreover we assume that $i m f_{\#} \subset C_{*}(Y, B)$, im $i^{\prime} \subset C_{*}(X, A)$, im $l \subset C_{*}(X, A)$ are direct summands (cf. [2] lemma 5.4.).

$\star \star) C_{*}(X, X)=\mathbf{C}_{*}(X)$ is acyclic.

$\star \star \star) C_{*}(\emptyset)=0$.

D4) To each homotopy $H: f_{0} \simeq f_{1}:(X, A) \longrightarrow(Y, B)$ there exists a natural, with $i^{\prime}$ and $l$ compatible chain homotopy

$$
D(H): C_{*}\left(f_{0}\right) \simeq C_{*}\left(f_{1}\right) .
$$

We call $C_{*}\left(\right.$ or $\left.\mathbf{C}_{*}\right)$ equipped with these additional structures

$$
\mathbf{C}_{*}=\left\{C_{*}, C_{*}^{\prime}, i^{\prime}, l, \varphi, \kappa\right\}
$$

a chain functor.

The derived homology $H_{*}\left(\mathbf{C}_{*}\right)$ of a chain functor is defined by

$$
H_{*}\left(\mathbf{C}_{*}\right)(X, A)=H_{*}\left(C_{*}(X, A)\right)
$$

with boundary

$$
\partial: H_{n}\left(\mathbf{C}_{*}\right)(X, A) \longrightarrow H_{n-1}\left(\mathbf{C}_{*}\right)(A)
$$

defined by the boundary $\bar{\partial}$ of $(\bar{S})$.

Moreover we will always assume that all functors $C_{*}$ have compact carriers, i.e. $1)$ to every $c \in C_{*}(X, A)$ there exists a $f:(K, L) \longrightarrow(X, A),(K, L)$ compact, $c^{\prime} \in C_{*}(K, L)$, such that $f_{\#}\left(c^{\prime}\right)=c$ and 2) if $f:(K, L) \longrightarrow(X, A), \quad(K, L)$ compact, $c^{\prime} \in C_{*}(K, L), \quad f_{\#}\left(c^{\prime}\right)=0$, then there exists a factorization $f=f_{1} g, \quad g$ : $(K, L) \longrightarrow\left(K_{1}, L_{1}\right), \quad\left(K_{1}, L_{1}\right)$ compact, such that $g_{\#}\left(c^{\prime}\right)=0$.

A homology theory $h_{*}=\left\{h_{n}, \partial, n \in \mathbb{Z}\right\}$ is related to a chain functor $C_{*}$ (or $\mathbf{C}_{*}$ ) whenever $H_{*}\left(\mathbf{C}_{*}\right)$ and $h_{*}$ are isomorphic as homology theories (i.e. the isomorphisms are natural and commute with boundaries). 
If in addition the derived homology $\left\{H_{n}\left(\mathbf{C}_{*}\right), \partial\right\}$ of a chain functor is isomorphic to a homology theory on $\mathfrak{K}^{2}$, then we call $C_{*}$ (or alternatively $\mathbf{C}_{*}$ ) a regular chain functor.

Compact carriers for the chain functors imply the same property for the derived homology theories.

Since $H_{*}\left(\mathbf{C}_{*}\right)$ satisfies all properties of a homology theory except possibly the excision axiom, we can formulate:

4.1. Definition: 1) A chain functor $\mathbf{C}_{*}=\left\{C_{*}, C_{*}^{\prime},, i^{\prime}, l, \varphi, \kappa\right\}$ is regular whenever the derived homology satisfies an excision axiom (i.e. $H_{*}\left(\mathbf{C}_{*}\right)$ is a homology theory).

2) Suppose $C_{*}, C_{*}^{\prime}: \mathfrak{K}^{2} \longrightarrow \mathbf{c h}$ are functors (possibly without compact carriers) coming together with mappings $l, \varphi, \kappa, i^{\prime}$ satisfying D1)-D4), but a) not necessarily $\star)$ and $b) i^{\prime}, l$ are not any more required to be inclusions. Then we call $\mathbf{C}_{*}=\left\{C_{*}, C_{*}^{\prime}, l, \varphi, \kappa, i^{\prime}\right\}$ by an abuse of notation an irregular-chain-functor.

Notice that an irregular-chain-functor may not be a chain functor at all. In [4] an irregular-chain-functor is called a weak chain functor. Although every irregularchain-functor allows the formation of derived homology groups, one does not necessarily have a boundary operator.

Summarizing we state that the class of chain functors contains the class of regular chain functors, while irregular-chain-functors form a much bigger class.

We record the following results on chain functors:

1) Every homology theory on a category of topological spaces (satisfying some mild conditions) is isomorphic to the derived homology of a chain functor (theorem 8.1. in $[2])$.

This chain functor is clearly regular but not uniquely determined by this property, although the procedure described in [2] is functorial. The question of its uniqueness is the subject of the present paper.

The properties required of a category of topological spaces such that theorem 8.1. in [2] holds are in fact very mild. So it turns out that the existence proof of a chain functor for a given homology theory carries over from e.g. the category of CW-pairs to the category of $C W$-spectra (i.e. to pairs of objects in the Boardman category). This is crucial for exploring localizations in [1].

2) The derived homology of a chain functor $H_{*}\left(\mathbf{C}_{*}\right)$ is always exact and homotopy invariant by definition, but does not necessarily satisfy an excision axiom. So only for regular chain functors $H_{*}\left(\mathbf{C}_{*}\right)$ is a generalized homology theory.

3) Every regular chain functor $\mathbf{C}_{*}$ being defined on the category of $\mathrm{CW}$-pairs gives rise to a $\mathrm{CW}$-spectrum $\left|\mathbf{C}_{*}\right|$ functorially such that

$$
H_{*}\left(\mathbf{C}_{*}\right) \approx\left|\mathbf{C}_{*}\right|_{*}
$$

denoting by $\mathbf{E}_{*}$ the homology theory associated with the spectrum $\mathbf{E}$ ) (cf. theorem 1.1. in [3]).

4.2. Definition 1) Let $\mathbf{K}_{*}, \mathbf{L}_{*}$ be two regular chain functors, then a mapping (or transformation) of chain functors

$$
\lambda: \mathbf{K}_{*} \longrightarrow \mathbf{L}_{*}
$$


is a natural transformation of functors

$$
\lambda_{(X, A)}: K_{*}(X, A) \longrightarrow L_{*}(X, A)
$$

such that $\lambda \mid K_{*}^{\prime}=\lambda^{\prime}$ is a natural transformation of functors

$$
\lambda^{\prime}: K_{*}^{\prime} \longrightarrow L_{*}^{\prime}
$$

which is compatible with $l, i^{\prime}$ and the natural chain homotopies $D(H)$ in D4).

2) Let $\mathbf{K}_{*}, \mathbf{L}_{*}$ be irregular, then a transformation of irregular-chain-functors $\lambda$ : $\mathbf{K}_{*} \longrightarrow \mathbf{L}_{*}$ is a pair of natural transformations

$$
\begin{aligned}
& \lambda: K_{*} \longrightarrow L_{*} \\
& \lambda^{\prime}: K_{*}^{\prime} \longrightarrow L_{*}^{\prime}
\end{aligned}
$$

commuting with $i^{\prime}, l$ and the chain homotopies $D(H)$.

3) Two irregular-chain-functors $\mathbf{K}_{*}, \mathbf{L}_{*}$ are equivalent as irregular-chain-functors whenever there exist transformations of chain functors

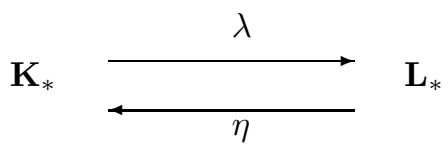

such that

$$
\lambda \eta=1_{L_{*}}, \eta \lambda=1_{K_{*}}, \lambda^{\prime} \eta^{\prime} \simeq 1_{L_{*}^{\prime}}, \eta^{\prime} \lambda^{\prime} \simeq 1_{K_{*}^{\prime}} .
$$

Observe that two regular chain functors are equivalent as chain functors, (i.e. one has transformations $(\star)$ satisfying $\lambda \eta=1_{L_{*}}, \eta \lambda=1_{K_{*}}$ ) if and only if they are equivalent as irregular-chain-functors. In case of irregular-chain-functors $\lambda^{\prime}$ is not a restriction of $\lambda$ to $K_{*}^{\prime}$.

It turns out that for a regular chain functor $H_{*}\left(\mathbf{C}_{*}\right)=\left\{H_{n}\left(\mathbf{C}_{*}\right), \partial\right\}$ is a homology theory on $\mathfrak{K}^{2}$ ([2] proposition A6) and that any transformation $\lambda: \mathbf{K}_{*} \longrightarrow \mathbf{L}_{*}$ between regular chain functors induces a natural transformation of homology theories. The latter follows because we have for any $z \in Z_{n}\left(C_{*}(X, A)\right)$

$$
z \sim l z^{\prime}+q_{\#}(a)
$$

and by definition

$$
\partial[z]=\left[i^{-1} d z^{\prime}\right] .
$$

Since $\lambda$ commutes with $l$ and $i^{\prime}$, we have

$$
\partial \lambda_{*}[z]=\lambda_{*} \partial[z] \cdot-
$$

Let

$$
\lambda_{1}, \lambda_{2}: \mathbf{K}_{*} \longrightarrow \mathbf{L}_{*}
$$

be two transformations of chain functors. They are homotopic whenever there exists a natural homotopy $D: \lambda_{1} \simeq \lambda_{2}$ which respects the subfunctors $\mathbf{K}^{\prime}{ }_{*}, \mathbf{L}^{\prime}{ }_{*}$ and commutes with $l$ and $i^{\prime}$. 
Consequently $\left[\mathbf{K}_{*}, \mathbf{L}_{*}\right]$ denotes the family of all homotopy classes of chain functor transformations, furnishing the homotopy category of chain functors.

One can define suspensions of chain functors as for chain complexes (simply by raising the dimension index). This gives rise to a triangulation (cf. [7] ) of the category of chain functors $\mathfrak{C} h$, allowing us to talk about exact sequences.

In [1] 2 we need:

D1') By changing $\varphi$ on $i m i^{\prime}$ we can assume that

$$
\varphi i^{\prime}=i \text {. }
$$

In particular we obtain for any $a \in C_{*}(A)$ :

$$
\kappa \varphi i^{\prime}(a)=i^{\prime}(a) \text {. }
$$

Proof: We are entitled to redefine $\varphi$ on the direct summand $i m i^{\prime} \subset C_{*}(X, A)$ (cf. [2] lemma 5.4.) without changing the chain functor within its isomorphism class. So we do this by setting $\varphi i^{\prime}=i$. All other properties of the given chain functor (e.g. $j_{\#} \varphi \simeq l, \varphi \kappa \simeq 1$ ) remain unchanged.

D1") Let $z^{\prime} \in C_{*}^{\prime}(X, A), d z^{\prime} \in i m i^{\prime}$ be a relative cycle then there exist a chain homotopy

$$
l \kappa \varphi\left(z^{\prime}\right)-l\left(z^{\prime}\right)=d \bar{D}\left(z^{\prime}\right)-\bar{D}\left(d z^{\prime}\right)
$$

with $\bar{D}\left(d z^{\prime}\right)=0$.

Proof. There are three chain homotopies:

$$
l \kappa \varphi \stackrel{\tilde{D}(\kappa \varphi)}{\simeq} j_{\#} \varphi \kappa \varphi \stackrel{j_{\#} D(\varphi)}{\simeq} j_{\#} \varphi \stackrel{\tilde{D}}{\simeq} l,
$$

with $\bar{D}\left(d z^{\prime}\right) \in C_{*+1}(X, A)$. However on $i m i^{\prime}$ we have

$$
l \kappa \varphi i^{\prime}(a)-l i^{\prime}(a)=l i^{\prime}(a)-l i^{\prime}(a)=0 .
$$

We alter $\bar{D}$ on $i m i^{\prime}$ to zero, without affecting the isomorphism class of $\mathbf{C}_{*}$.-

A natural transformation $\lambda: \mathbf{K}_{*} \longrightarrow \mathbf{L}_{*}$ is supposed to commute with $i^{\prime}, l$, and $D(H)$. We do not require explicitely that $\lambda$ commutes with $\kappa, \varphi$ and all the other items of a chain functor (like the chain homotopies $\varphi \kappa \simeq 1, j_{\#} \varphi \simeq l$ ). However this would not be such a severe restriction:

4.3. Definition: A natural transformation of chain functors is called geometric ( $A$ g-transformation) whenever im $\lambda \subset L_{*}()$ is a direct summand.

We have:

4.4. Proposition: If $\lambda: \mathbf{K}_{*} \longrightarrow \mathbf{L}_{*}$ is a g-transformation, then there exists a g-transformation $\tilde{\lambda}: \mathbf{K}_{*} \longrightarrow \tilde{\mathbf{L}}_{*}$, commuting with all structure of chain functors (e.g. $\varphi, \kappa, \cdots)$ and an equivalence $\varepsilon: \tilde{\mathbf{L}}_{*} \approx \mathbf{L}_{*}$ such that

$$
\varepsilon \tilde{\lambda}=\lambda \text {. }
$$


Proof: $i m \lambda \subset L_{*}()$ is a direct sumund and free. So we are allowed to change $\varphi, \kappa$, as well as the relevant homotopies first on free generators of $i m \lambda$ afterwards extending this linearily on whole $i m \lambda$ so that $\lambda$ now commutes with all these items. This establishes $\tilde{\mathbf{L}}_{*}$. Since none of these mappings and chain homotopies is natural, this can be done without changing the isomorphism class of $\mathbf{L}_{*}$. The equivalence $\varepsilon$ is the identity as a functor transformation (forgetting the additional structure of a chain functor).-

Moreover we can assert that every transformation $\lambda: \mathbf{K}_{*} \longrightarrow \mathbf{L}_{*}$ is up to a homotopy equivalence a $\mathrm{g}$ - transformation:

4.5. Proposition: Let $\lambda: \mathbf{K}_{*} \longrightarrow \mathbf{L}_{*}$ be a transformation of chain functors, then there exists a homotopy equivalence $\gamma: \mathbf{L}_{*} \longrightarrow \tilde{\mathbf{L}}_{*}$ and a g-transformation $\tilde{\lambda}: \mathbf{K}_{*} \longrightarrow \tilde{\mathbf{L}}_{*}$ such that

$$
\tilde{\lambda}=\gamma \lambda
$$

Proof: We establish $\tilde{\mathbf{L}}_{*} \supset \mathbf{L}_{*}$ by "doubling $i m \lambda$ ":

Let $\Lambda$ be a second copy of $i m \lambda \subset L_{*}()$. To each $c \in i m \lambda$ we find a $\tilde{c} \in \Lambda$. If $w$ is any of the operations $\varphi, \kappa$ or the relevant chain homotopies, we define

$$
w(\tilde{c})=\left\{\begin{array}{lll}
\widetilde{w(c)} & \cdots & w(c) \in i m \lambda \\
w(c) & \cdots & w(c) \notin i m \lambda .
\end{array}\right.
$$

Then we invent new connecting chains $v_{c}, c \in i m \lambda$ satisfying

$$
d v_{c}+v_{d c}=c-\tilde{c}
$$

and formally $v_{c}=0$ for all $c \notin i m \lambda$. For any $w$ as before, we set $w\left(v_{c}\right)=v_{w(c)}$. All this is firstly performed for free generators and then for any $c \in i m \lambda$ by linear extension, providing us with an enlarged chain functor $\gamma: \mathbf{L}_{*} \subset \tilde{\mathbf{L}}_{*}, \gamma(c)=\tilde{c}$, with $\tilde{\mathbf{L}}_{*}=\mathbf{L}_{*} \oplus\left\langle\Lambda,\left\{v_{c}\right\}\right\rangle$ being the amalgameted sum. The inclusion $\gamma$ is obviously a chain homotopy equivalence. In particular the inclusion turns out to induce an isomorphism in homology. We define

$$
\tilde{\lambda}(a)=\widetilde{\lambda(a)},
$$

obtaining a g-transformation with

$$
\tilde{\lambda}=\gamma \lambda \text { - }
$$

Remarks: 1) In order to obtain D1') and D1") we only need to know that $i m i^{\prime}$ is a direct summand in $C^{\prime}(X, A)$, therefore it would suffice to require only this. However the chain functor constructed in [2] being associated with a given homology theory satisfies automatically all the other properties listed in $\star$ ) (cf. [2] lemma 5.4). It turns out that verifying all these properties together for chain functors, which will be constructed does not cause any trouble at all.

The essence of D1'), D1") is that these properties can be assumed to be true (up to an equivalence of chain functors) for any chain functor.

2) Suppose that the free generators of $K_{*}()$ as well as those of $L_{*}()$ are geometrically defined (as for example singular simplexes for ordinary singular homology or singular manifolds for bordism theories are), then a transformation is a g-transformation, 
whenever generators are transformed into generators by all operations in a chain functor.

3) Denoting the category of chain functors by $\mathfrak{C h}$ we pointed out already, that, according to 4.5 ., every $\lambda \in \mathfrak{C h}\left(\mathbf{K}_{*}, \mathbf{L}_{*}\right)$ is, up to a homotopy equivalence, a gtransformation which, according to 4.4. can be assumed to commute with all conceivable structure of a chain functor.

Finally we would like to asses how restrictive the assumption is that a chain functor is required to have a natural basis (i.e. that base elements are mapped into base elements by continuous mappings). In [2] lemma 5.4. 2) this is guaranteed for the chain functor, which is established there.

Suppose $\mathbf{C}_{*}=\left\{C_{*}, C_{*}^{\prime}, i, l i^{\prime}\right\}$ satisfies all asumptions of a chain functor, but $C_{*}(X, A)$ is not necessarily free at all.

4.6. Proposition: There exists a chain functor $\mathbf{K}_{*}$ (i.e. all $K_{*}(X, A)$ are free with a natural base) and a transformation $\lambda: \mathbf{K}_{*} \longrightarrow \mathbf{C}_{*}$ (a concept, which is defined as in definition 4.2.) inducing an isomorphism in homology.

Proof: Let $K_{*}^{(1)}(X, A)$ be the free group, generated by the non-zero elements of $C_{*}(X, A)$. Let $k=\sum m_{i}\left[c_{i}\right]$ be an element of $K_{*}^{(1)}(X, A)$ then there exists an associated $k^{(1)}=\left[\sum m_{i} c_{i}\right] \in K_{*}^{(1)}(X, A)$. We invent a connecting chain $w_{k}$ satisfying

$$
d w_{k}+w_{d k}=k-k^{(1)}
$$

and for continuous $h:(X, A) \longrightarrow(Y, B)$

$$
h_{\#}\left(w_{k}\right)=\left\{\begin{array}{lll}
w_{h_{\#}(k)} & \cdots & k^{(1)} \neq 0 \\
0 & \cdots & k^{(1)}=0
\end{array}\right.
$$

Let $\mathbf{K}_{*}^{(2)}$ be $\mathbf{K}^{(1)}$ amended by these new chains ( as in the proof of 4.5.) and set $\mathbf{K}_{*}=\mathbf{K}_{*}^{(2)} \cup$ cone $W_{*}$ with $W_{*}$ being generated by all connecting chains $w$. This can easily be equipped with the structure of a chain functor: We define e.g. $\varphi(k)=\sum m_{i}\left[c_{i}\right]$, and $\varphi\left(w_{k}\right)=0$. The mapping $\lambda: \mathbf{K}_{*} \longrightarrow \mathbf{C}_{*}$ is defined by $[c] \longmapsto c$ and $\lambda\left(w_{k}\right)=0$. It clearly induces an isomorphism of the homology groups.

\section{Motivation of the definition of a chain functor}

Let $h_{*}=\left\{h_{n}, \partial\right\}$ be a generalized homology theory, defined on some $\mathfrak{K}^{2}$. It is desirable to have some kind of a chain theory available, providing us with chains, cycles and individual homologies between these cycles, furnishing us with the correct homology groups $h_{*}(X, A)$ as well as with the correct boundary operator $\partial: h_{n}(X, A) \longrightarrow h_{n-1}(A)$. Examples are ordinary singular or Steenrod-Sitnikov homology theories, but also bordism theories, where the chains are represented by singular manifolds with boundaries, resp. the cycles by closed singular manifolds.

To this end one would like to detect a functor $C_{*}: \mathfrak{K}^{2} \longrightarrow \mathbf{c h}$ and a natural isomorphism

$$
\lambda: H_{*}\left(C_{*}(X, A)\right) \approx h_{*}(X, A) .
$$


Moreover we require the existence of a short exact sequence

$$
0 \longrightarrow C_{*}(A) \stackrel{i_{\#}}{\longrightarrow} C_{*}(X) \stackrel{j_{\#}}{\longrightarrow} C_{*}(X, A) \longrightarrow 0
$$

such that the boundary $\bar{\partial}$ defined by $(7)$ is compatible with the boundary $\partial$ of $h_{*}$, i.e. such that the diagram

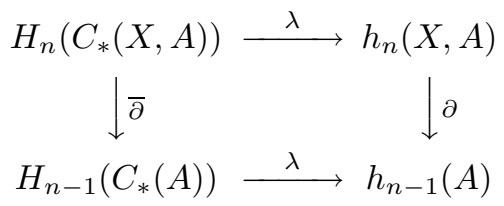

is commutative.

The great disappointment comes with a theorem of the authors of [6] asserting that the existence of such a naive chain theory, satisfying (6) - (8) is equivalent to the fact that (on the category of CW-pairs) $h_{*}$ is the direct sum of ordinary homology theories.

Since most homology theories (as for example many bordism theories) are not of this type, the existence of such a chain theory can not be expected.

However since there is nevertheless still need for some kind of a chain theory for arbitrary homology theories, one has to modify (6)-(8) appropriately.

This is the reason for introducing chain functors in $\S 4$. The short exact sequence (5) is replaced by the diagram $(\bar{S})$. The "relative cycles" (i.e. the chains $c \in C_{*}(X)$ satisfying $\left.d c \in i m i_{\#}\right)$ are retrieved by the chains $z^{\prime} \in C_{*}^{\prime}(X, A)$ with $d z^{\prime} \in i m i^{\prime}$.

As already indicated in $\S 4$, the main interest in chain functors originates from the fact that every homology theory $h_{*}()$ is associated with a chain functor $\boldsymbol{K}_{*}$, which means that

$$
H_{*}\left(\boldsymbol{K}_{*}\right) \approx h_{*}() .
$$

A convenient way to prove this (not the original proof given in [2]) is to deal firstly with homology theories $h_{*}($ ) having a classifying spectrum $\boldsymbol{E}$, i.e.

$$
h_{*}() \approx \boldsymbol{E}_{*}()=\pi_{*}(\boldsymbol{E} \wedge()) .
$$

The relevant statement is therefore:

There exists a functor

$$
\Phi: \text { Spec } \longrightarrow \mathfrak{C h},
$$

Spec being the category of spectra, together with an isomorphism of homology theories

$$
H_{*}\left(\Phi(\boldsymbol{E})() \approx \boldsymbol{E}_{*}()\right.
$$

which is natural in all variables .

The first step to verify this is to establish the free chain complex functor $F_{*}^{1}: \mathfrak{K}^{2} \longrightarrow$ $\boldsymbol{c h}$, with stable mappings $f: D^{n} \longrightarrow \boldsymbol{E} \wedge X$ (i.e. one has $f=\left\{f_{k}: D^{n+k} \longrightarrow\right.$ $\left.E_{k} \wedge X\right\}$ ). The boundary $d f$ is established by forming $f \mid b d D^{n+k}$. This $F_{*}^{1}$ is far from having the desired homology and must be further adapted, which causes some technical problems. 
The proof of this (seemingly less general) assertion can be used for a proof of the more general fact that any homology theory $h_{*}$ ( ) (eventually without a classifying spectrum) is associated with a chain functor. There exists a key to translate the arguments for verifying the restrictive assertion, to the general case.

The fact that $\varphi_{\#}, \kappa_{\#}$ as well as the chain homotopies in $\left.\S 4 \mathrm{D} 1\right)$ are not always natural is not accidental:

Call to this end a chain functor $\mathbf{C}_{*}$ flat whenever all these items are natural, while a homology theory $h_{*}$ (defined on any category of pairs of spaces) is called flat whenever there exists a (naive) chain theory (6)-(8) associated with $h_{*}$. Hence, according to the main result of [6], on CW-pairs $h_{*}$ is flat, whenever $H_{*}$ is the direct sum of ordinary homology theories.

It turns out that (for any $\left.\mathfrak{K}^{2}\right)$ ([5] theorem 3.3.):

$h_{*}$ is flat, if and only if there exists a flat chain functor associated with $h_{*}$.

As a result the lack of naturality of $\varphi_{\#}, \kappa_{\#}, \cdots$ is closely related to the non-vanishing of the Postnikov-invariants of the classifying spectrum $\mathbf{E}$ of a homology theory.

In dealing with bordism homology $\Omega_{*}($ ) one has a completely different option to detect a chain functor $\boldsymbol{K}_{*}$ with

$$
H_{*}\left(\boldsymbol{K}_{*}\right)() \approx \Omega_{*}():
$$

The candidates for the chains are now singular manifolds and the cycles closed singular manifolds (cf. [5] for further references).

Neither the present paper nor [1] is attempting to treat $\mathfrak{C} h$ as a closed model category or as a triangulated category, incorporating it in the list of candidates for a stable homotopy category, as this is now the custom. This is postponed to a forthcoming paper. Our present approach uses chain functors entirely as a concept, for establishing results about stable homotopy theory for spectra.

However with the introduction of irregular-chain-functors, the category $\mathfrak{C} h$ becomes "larger" than the Boardman category, providing new objects, which can not be realized as spectra, although they are needed for a systematic description of some phenomena in stable homotopy theory, as will become clear in the course of [1]. 


\section{References}

[1] F. W. Bauer, Tensor products of spectra and localizations

[2] - "-, Generalized homology theories and chain complexes Annali di Matem. pura ed appl. (IV) Vol.CLV (1989) 1143-1191

[3] - - , Classifying spectra and generalized homology theories Annali di Matem. pura ed appl. (IV) Vol.CLXIV (1993) 365-399

[4] - "-, A strong homology theory satisfies a clusteraxiom Note di Matematica, Vol. X, Suppl. n.1, (1990) 73-102

[5] - " - , The Boardman category of spectra, chain complexes and (co)localizations Homology, Homotopy and Applications Vol. 1 No. 3 (1999) 95 116

[6] R. O. Burdick, P. E. Conner, E. E. Floyd Chain theories and their derived homology Proceedings of the AMS, 19 (1968) 1115- 1118

[7] H. R. Margolis, "Spectra and the Steenrod Algebra" North-Holland Mathem. Library, Amsterdam-NewYork-Oxford (1983)

[8] R. Switzer, "Algebraic Topology - Homotopy and Homology" Springer, BerlinHeidelberg-NewYork (1975)

This article may be accessed via WWW at http://www.rmi.acnet.ge/hha/ or by anonymous ftp at

ftp://ftp.rmi.acnet.ge/pub/hha/volumes/2001/n2/n2.(dvi,ps,dvi.gz,ps.gz)

Friedrich W. Bauer f.w.bauer@mathematik.uni-frankfurt.de

Johann-Wolfgang Goethe Universität

Fachbereich Mathematik

Robert-Mayer Strasse 8-10

60054 Frankfurt a. M.

Germany 